\title{
Biotechnological investigation for the prevention of biofouling. I. Biological and biochemical principles for the prevention of biofouling
}

\author{
Sibylle Abarzua, Sabiene Jakubowski
}

Universität Rostock, Fachbereich Biologie, Lehrstuhl Biochemie, Doberaner Straße 143, D-18051 Rostock, Germany

\begin{abstract}
The most important biological and biochemical methods with potential for the prevention of biofouling are described. Among these methods, the isolation of biogenic agents produced by several species of micro- and macroalgae and marine invertebrates with antibacterial, antialgal, antiprotozoan and antimacrofouling properties may be the most promising and effective method for the prevention of biofouling. The isolated substances with the most potent antifoulant activity are fatty acids, terpenes, terpenoids, lipoproteins, glycolipids, phenols, lactons, peptides and steroids. The advantage of the utilization of micro- and macroalgae for the isolation of biogenic agents is that algae can be cultivated in a short time in mass culture, independent of season. Furthermore, they can be manipulated to a large extent in the direction of the 'production of biogenic agents' However, the cultivation of micro- and macroalgae is very expensive. Marine invertebrates must be collected in certain seasons. This collection of marine invertebrates could lead to an uncontrolled exploitation of marine organisms and to a change in the balance of marine ecosystems. Therefore, determination of the chemical structure and the subsequent synthesis of the determined biogenic agents is necessary if marine invertebrates are to be used as producers of biogenic agents. Antifouling systems must be both environmentally safe and effective for at least 3 yr when formulated as antifouling paints. There have been a few attempts at this, but no applicable successes have been reported to date.
\end{abstract}

KEY WORDS: Antifouling - Biofouling Growth inhibition Marine bioactive agents - Macrofoulers · Microfoulers · Settlement

\section{INTRODUCTION}

\section{What is biofouling?}

Biofouling is one of the most important problems currently facing marine technology. In the marine environment any solid surface will become fouled. Materials submerged in seawater experience a series of discrete physical, chemical and biological events which results in the formation of a complex layer of attached organisms known as biofouling.

Loeb \& Neihof (1975), Baier (1984) and Lewin (1984) have shown that the first event is the accumulation of an organic 'conditioning' film consisting of chemical compounds (mostly protein, proteoglycans and polysaccharides) making the surface wettable (Dexter
1978). This process occurs in the first minutes of the biological settlement (Fig. 1). After approximately 1 to $2 \mathrm{~h}$, the colonization of bacteria involving 2 distinct phases, a reversible approach phase ('adsorption') and a nonreversible attachment phase ('adhesion'), occurs (Marshall 1980, Wahl 1989) (Fig 1). The first, reversible adsorption, is an instantaneous attraction which holds bacteria near the surface. The bacterial adsorption is essentially governed by physical forces: Brownian motion, electrostatic interaction, gravity, and van-derWaal forces (Fletcher \& Loeb 1979, Walt et al. 1985). The phenomenon is termed reversible because the organisms can easily be removed before substantial contact of cell surface has been made. The second, irreversible attachment phase can be made by bacteria that produce extracellular bridging polymer (e.g. poly- 


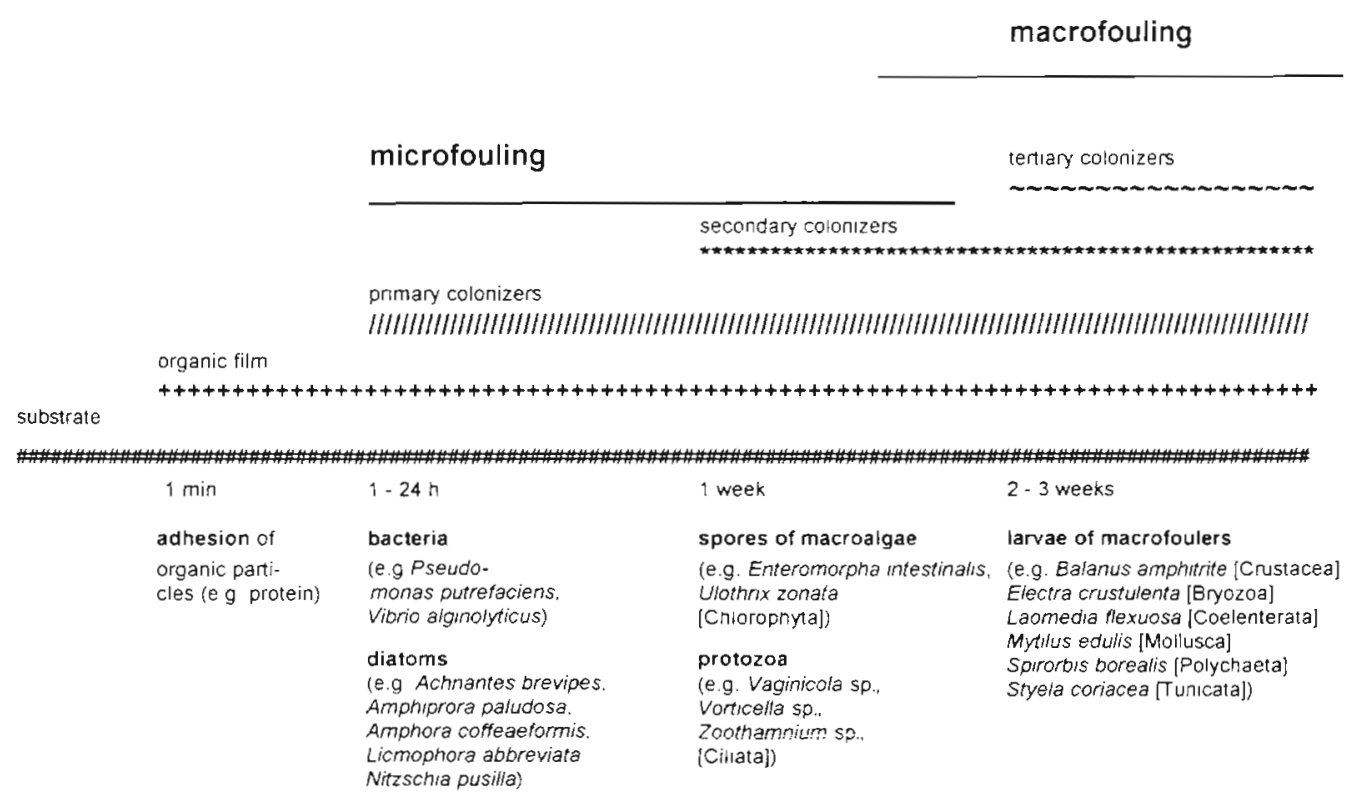

Fig. 1. Temporai structure of settlement saccharide fibrils consisting mostly of glucose and fruciusej. It is known that these polysaccharide fibrils (slimes) are anchored to their chemical counterparts in the macromolecular film by lectins or divalent cations $\left(\mathrm{Ca}^{++}, \mathrm{Mg}^{++}\right)$(Costerton et al, 1978). With the establishment of covalent bonds between the bacterial glycocalix and the macromolecular film the adsorption phase blends into the adhesion phase (Wahl 1989). The growing bacterial lawn, composed of dead and living cells and their secreted 'slime', together with the macromolecular film, constitutes the so-called primary film (slime film) (Wahl 1989).

Diatoms, spores of macroalgae and protozoa appear after the development of the primary film (Fig. 1) with a clear quantitative dominance of the diatoms (Marshall et al. 1971, Caron \& Sieburth 1981, Cuba \& Blake 1983, Zahuranec 1991). Benthic diatoms are attached by mucus secretion (Cooksey et al. 1984, Ferreira \& Seeliger 1985) and may densely cover wide substratum areas. While in the majority of observed events diatom colonization was always preceded by bacterial attachment (Little 1984), there may be exceptions (Sieburth \& Tootle 1981, Maki et al. 1988). Attachment of spores of macroalgae is realized by species such as Enteromorpha intestinalis and Ulothrix zonata; protozoan colonizers belong mostly to the sessile or hemisessile forms (e.g. Ciliata) or are mobile predators of microorganisms, not being considered to be true epibionts.

Bacteria and diatoms represent the primary colonizers and spores of macroalgae and protozoa constitute the secondary colonizers in the process of microfouling (Fig. 1). A clear separation between microfouling and macrofouling is impossible because the spores of macroalgae belong to the macrofouling organisms
(Von Oertzen et al. 1989), therefore there is an overlapping between micro- and macrofouling (Fig. 1). Larvae of macrofoulers (sessile marine organisms such as tunicates, coelenterates, bryozoans, barnacles, mussels, polychaetes), which are called tertiary colonizers, then attach to the microfouling film (Fig. 1) (Takazawa et al. 1992). Larvae of macrofoulers prefer to settle on surfaces coated with microbial and algal films (Colwell 1983).

According to micro- and macrofouling processes the following overlapping time sequence is observed: bacteria appear after approximately 1 to $2 \mathrm{~h}$, diatoms after $24 \mathrm{~h}$, spores of macroalgae and protozoa after $1 \mathrm{wk}$ and larvae of macrofoulers after 2 to $3 \mathrm{wk}$ (Von Oertzen et al. 1989). It seems that all micro- and macrofoulers produce adhesive substances necessary for their attachment to solid surfaces such as the hull of a ship (Cooksey et al. 1984). In the literature there are contradictory opinions on the question whether this sequence of events constitutes a real ecological succession or not (Little 1984).

The settlement of micro- and macrofoulers on the hulls of ships must be prevented for both economic and ecological reasons. The bacterial slime films and the large numbers of barnacles, mussels and tunicates which accumulate on ships increase drag forces and surface corrosion, thereby causing additional fuel, $\mathrm{CO}_{2}$ emissions and maintenance costs (Gitlitz 1981)

\section{Effect of the utilization of antifouling paints}

In the efforts to avoid marine biofouling, antifouling paints are used, mostly with copper and tri-n- 
butyltin (TBT) as very effective active agents (Willemsen \& Ferrari 1993). The antifouling paints prevent biofouling by releasing effective biocides at a constant rate. Since the early 1970s, triaryltin and trialkyltin compounds have been increasingly used in antifouling paints because of their excellent ability to prevent marine organisms from becoming encrusted on ship bottoms and culturing nets (Suzuki et al 1992).

TBT is used as a biocide in coatings in 3 different ways: in free association paints (biocide dispersed in a resinous matrix), in ablative paints (biocide is bonded in a less permeable matrix that gradually flakes off) and in self-polishing copolymers (SPC) (biocide is chemically bonded). Self-polishing organotin copolymer formulations have the best release rate characteristics of currently available antifouling paints and are capable of maintaining vessels free from macroscopic biofouling for periods of up to $5 \mathrm{yr}$ (Christie \& Dalley 1987). In use as an antifouling additive the use of marine paints containing the broad-spectrum poison TBT grew to an estimated $136000 \mathrm{~kg} \mathrm{yr}^{-1}$ by the late 1980s (Uhler et al. 1993). The worldwide application of TBT-based paints has caused a growing pollution of the environment and foods on a worldwide scale (Suzuki et al. 1992). TBT harms many forms of marine life other than fouling organisms, including economically important species like oysters. Thus, shell deformation of the Pacific oyster Crassostrea gigas, and little or no natural oyster larvae settlement on hard substrates, suggesting toxic effects in early life stages, were obseved in Arcachon Bay, France. More extreme deformations occur in the common dogwhelk Nucella lapillus, a species of thick-shelled snail found around the southwest peninsula of England. The occurrence of imposex (the development of male characteristics, notably a penis and a sperm duct, on females) was shown in centres of boating and shipping activities, which is a sign of decline (Oehlmann et al. 1993). Fewer females occur than would be expected, and juveniles and deposited egg capsules are scarce or absent, indicating a low reproductive capacity.

Due to risk in application of organotin antifouling coatings they have come under increasing governmental regulation in the United States and many western European countries (Price et al. 1992). This has initiated further development of TBT-free antifouling paints, containing herbicides, antibiotica, copper salts and other organic additives like amino-, azine- and thio-derivatives (Golchert 1993, Peterson et al. 1993). These TBT-free antifouling coatings are capable of maintaining ships free from biofouling for about 3 yr but none of these additives can compete with organotin containing SPCs (Golchert 1993).

\section{Alternative methods for the prevention of biofouling}

Due to present and expected future restrictive regulation on the use of TBT (Dalley 1987) and probably other polluting antifouling compounds there is a growing need for other methods of the prevention of biofouling. Several physical/mechanical, physical/chemical and biological/biochemical principles for the prevention of biofouling were used in the last $30 \mathrm{yr}$ (Gerencser et al. 1962, Schulz \& Subklev 1964, Kühl \& Neumann 1969, Loeb \& Neihof 1975, Characklis 1981, Dhar et al. 1981, Branscomb \& Rittschof 1984, Fletcher \& Baier 1984, Humphries et al. 1986).

It is the aim of the present work to describe and evaluate the most important biological/biochemical methods used for the prevention of settlement and to discuss the possibility of using biogenic agents produced by several marine organisms with antibacterial, antialgal, antiprotozoan and antimacrofouling properties for the prevention of biofouling.

\section{BIOLOGICAL AND BIOCHEMICAL METHODS FOR THE PREVENTION OF BIOFOULING}

\section{Dissolution of adhesive substances by several enzymes}

All biofouling organisms achieve their attachment by using adhesive substances, the chemical structure of which is quite similar for bacteria, diatoms, spores of macroalgae and macrofoulers (acid polysaccharides and glycoproteins) (Fletcher \& Floodgate 1973, Haug 1976, Humphrey et al. 1979, Percival 1979, Daniel et al. 1987, Wigglesworth-Cooksey \& Cooksey 1992). It is assumed that a dissolution of these substances would reduce the long-term attachment to solid surfaces (Christie et al. 1970, Dempsey 1981, Evans 1981, Cinti et al. 1987). Certain enzymes, like trypsin, chymotrypsin, pronase and $\alpha$-amylase, are active in the weakening action on adhesion in the bacterium Vibros proteolytica and in zoospores of the green macroalga Enteromorpha intestinalis (Christie et al. 1970, Paul \& Jeffrey 1985). Short-term exposure to the enzymes actinidin and pepsin can be used to separate the stalked epiphytic diatoms Synedra tabulata and Licmophora species from their brown or green algal hosts (Booth 1981). A variety of hydrolytic enzymes were tested for effects on barnacle settlement on solid surfaces (Rittschof et al. 1991). The majority of enzymes tested (cellulase, chitinase, collagenase, trypsin, chymotrypsin, carboxypeptidase A, B, Y) had little effect on settlement. But protease XI significantly inhibited the settlement of barnacles on polystyrene and glass surfaces, while papain had an inhibitory 
effect on polystyrene surfaces only. The biotechnological production of these enzymes in large quantities is possible, but relatively expensive. Furthermore, the enzymes are not permanently stable and different enzymes are necessary to split the various adhesive substances. Therefore, the method of dissolution of adhesive substances is applied only partly in practice.

\section{Intervention in the metabolism of fouling organisms}

A well-balanced supply of calcium is necessary for a successful adhesion of bacteria, diatoms and spores of macroalgae (Marshall et al. 1971, Grant et al. 1973, Haug 1976, Fletcher 1979, Cooksey \& Cooksey 1980, Cooksey 1981, Turakhia \& Characklis 1989). The process of synthesis and secretion of adhesive substances leading to motility and adhesion in diatoms can be prevented by uncouplers of energy metabolism (CCCP, carbonyl cyanide 3-chlorophenyl hydrazone), protein synthesis inhibitors (cycloheximide), and compounds that interfere with Ca transport (D-600, $\alpha$-isopropyl-a-[i(N-meinyi-iv-homoveratryl)- $\gamma$-amino-propyl]3,4-5-trimethoxy phenylacetonitrile) (Cooksey \& Cooksey 1980, Cooksey 1981, Cooksey et al. 1984). The formation of sulfated polysaccharides responsible as gel for the attachment of the spores of green macroalgae can be blocked by the reduction of calcium and borate supply (Haug 1976). All these experiments, however, have been carried out only under laboratory conditions and without regard to antifouling aspects.

\section{Competitive inhibition of receptors by offering specific lectin-like substances}

It has been shown for many macrofouling organisms that special substances (e.g. insoluble protein-conjugates) can contact with corresponding receptors of the larvae and induce an attachment and a metamorphosis. By offering specific lectin-like substances, which have a stronger affinity to the receptors than the insoluble protein-conjugates, the attachment is affected (Morse 1984). It is also possible to inhibit adhesion processes by offering simple sugars to bacterial lectins (reversed process) (Corfield \& Schauer 1982, Reuter et al. 1982, Imam et al. 1984, Sönnichsen 1993). These experiments have been performed only under laboratory conditions.

\section{Negative chemotaxis}

Numerous experimental results prove that special organic nontoxic substances (e.g acrylamide, benzoic, tannic and sialic acids) have a negative effect on the chemotaxis of bacteria, which results in an inhibition of bacterial attachment (Chet et al. 1975, Mitchell et al. 1975, Chet \& Mitchell 1976). Chemotaxis acts as a form of gravity, holding motile bacteria close to biofouling surfaces (Mitchell \& Kirchman 1984). The strong negative charged molecules of sialic acid are able to hold bacteria at distance, so that fixed association with the surface is blocked up (Corfield \& Schauer 1982, Reuter et al. 1982, Sönnichsen 1993). Successful field experiments have not yet been carried out.

\section{Biogenic agents}

Many marine organisms produce biogenic agents with antibacterial, antialgal, antifungal, antiprotozoan and antimacrofouling properties to dcfend themselves against robbers and settlement in the marine environment. Therefore, the production and isolation of biogenic substances from marine organisms seem to be the most promising and effective methods for the prevention of biofouling.

\section{BIOGENIC AGENTS AND THEIR EFFECTS ON FOULING ORGANISMS}

\section{Definition of biogenic agents}

Numerous living organisms, including microorganisms, fungi, plants and animals, are able to synthesize biogenic agents. These biogenic agents are synthesized in the secondary metabolism of the producer and are not directly essential for its life. They serve animals as protection from enemies (e.g. robbers), plants as protection against feeding and microorganisms in the suppression of growth and reproduction of other microbes. Teuscher \& Lindequist (1988) defined biogenic agents as follows: biogenic agents are chemical compounds which are synthesized by living organisms and which, if they exceed certain concentrations, cause temporary or permanent damage or even the death of other organisms by chemical or physicochemical effects. The concentration which causes damage and the extent of this damage are determined by the type of substance, the place and type of application, the duration of the effect, the individual sensitivity of the corresponding organism and other factors

Marine organisms (especially micro- and macroalgae and marine invertebrates) were investigated in the last decade with growing intensity regarding chemistry and pharmacology of their active agents. The sub- 
stances isolated in this connection belong, above all, to the groups of fatty acids, terpenes, terpenoids, lipoproteins, glycolipids, phenols, lactons, alkaloids and peptides. Depending on the screening methods used, the effects of the isolated potential drugs were mainly antibacterial, antiviral and antifungal. According to Ireland et al. (1993), ca 35\% of these biogenic compounds are produced by micro- and macroalgae and ca $65 \%$ by marine invertebrates. The ability of marine organisms to produce biogenic substances with antibacterial, antialgal, antiprotozoan and antimacrofouler properties could be used in the prevention of biofouling.

\section{Biogenic agents isolated from micro- and macroalgae}

An immense number of substances with antibacterial, antiviral, antifungal and pharmacological properties have been isolated from micro- and macroalgae, analyzed, and tested for medical purposes in the last few years. Tables 1 to 5 give an overview of the existence of special biogenic compounds in micro- and macroalgae and their effect on the growth of several bacteria, algae, fungi, protozoa and macrofoulers. Several biogenic compounds, such as bromophenols, malyngolides, aponin, cyanobacterin, hapalindoles, fischerellin, galactosyldiacylglycerols, tjipanazoles and scytophycins, isolated from special species of Cyanophyceae that have either antibacterial, anti- algal, antifungal or antiprotozoan effects, are given in Table 1. Macrofoulers were not tested. Tables $2 \& 3$ show bioactive substances (e.g. fatty acids, glycolipids/lipoproteins, terpenes/carbohydrates, goniodomin, chlorophyll $c$ and $\alpha$-linolenic acid) isolated from special species of Chrysophyceae, Dinophyceae and Chlorophyceae that have inhibitory effects on the growth of bacteria, algae, fungi and protozoa. Macroalgae like Phaeophyceae, Chlorophyceae, Conjugatophyceae and Charophyceae also produce biogenic substances with antibacterial, antialgal, antifungal, antiprotozoan and antimacrofouling effects (Tables 4 \& 5). The special biogenic substances listed in the tables were isolated from micro- and macroalgae and tested predominantly with regard to antibacterial, antialgal and antifungal activities and not with regard to antifouling aspects. Effects on the growth of macrofoulers (e.g. polychaetes, mussels) were investigated only with some species of macroalgae (Laminaria digitata, Costaria costatum, Undaria pinnatifida; Tables $4 \&$ 5). In contrast to micro- and macroalgae, higher plants are well documented as antifouling agents (Sawant et al. 1992, Sawant \& Wagh 1994).

The cultivation of micro- and macroalgae as well as the extraction of biogenic agents is expensive. However, algae can be used very well as 'extraction organisms' because they can be cultivated in a short time in mass culture independent of season and can be manipulated to a large extent in the direction of 'production of biogenic agents'.

Table 1. Existence and effects of biogenic agents isolated from Cyanophyceae (microalgae)

\begin{tabular}{|c|c|c|c|c|c|c|}
\hline \multirow[t]{2}{*}{ Species } & \multirow[t]{2}{*}{ Biogenic agent } & \multirow[b]{2}{*}{$\begin{array}{c}\text { Anti- } \\
\text { bacterial }\end{array}$} & \multicolumn{2}{|c|}{ Effects } & \multirow[b]{2}{*}{$\begin{array}{c}\text { Anti- } \\
\text { protozoan }\end{array}$} & \multirow[t]{2}{*}{ Source } \\
\hline & & & $\begin{array}{l}\text { Anti- } \\
\text { algal }\end{array}$ & $\begin{array}{l}\text { Anti- } \\
\text { fungal }\end{array}$ & & \\
\hline Calothrix brevissima & Bromophenols & $x$ & $x$ & & & Pedersen \& Da Silva (1973) \\
\hline Lyngbya majuscula & Malyngolide & $x$ & & & & Cardllina et al. (1979) \\
\hline Gomphosphaeria aponina & Aponin & & $x$ & & & Eng-Wilmot et al. (1979) \\
\hline Anabaena flos-aquae & $?$ & & & & $x$ & Snell (1980) \\
\hline Scytonema hofmanni & Cyanobacterin & & $x$ & & & $\begin{array}{l}\text { Mason et al. (1982), } \\
\text { Gleason \& Paulson (1984), } \\
\text { Gleason \& Baxa (1986) }\end{array}$ \\
\hline Fischerella muscicola & $?$ & & $\times$ & & & Flores \& Wolk (1986) \\
\hline Scytonema pseudohofmanni & Scytophycins & & & $×$ & & Ishibashi et al. (1986) \\
\hline Hapalosiphon fontinalis & Hapalindoles & & $\times$ & $x$ & & Moore et al. (1987) \\
\hline Synechocystis leopoliensis & Methanolic extracts & $x$ & & & & Cannell et al. (1988) \\
\hline Oscillatoria sp. & Ether extracts & & $x$ & & & Baghi et al. (1990) \\
\hline Fischerella muscicola & Fischerellin & & $x$ & & & Gross et al. (1991) \\
\hline Nostoc muscorum & Methanolic extracts & & & $x$ & & De Mule et al. (1991) \\
\hline Nostoc muscorum & Aqueous extracts & $x$ & & & & Bloor \& England (1991) \\
\hline Nostoc linckia & Cyanobacterin LU-1 & & $x$ & & & Gromov et al.(1991) \\
\hline Phormidium tenue & Galactosyldiacylglycerols & & $x$ & & & Murakami et al. (1991) \\
\hline Tolypothrix tjipanasensis & Tjipanazoles & & & $x$ & & Bonjouklian et al. (1991) \\
\hline Scytonema ocellatum & Tolytoxin (scytophycin) & & & $\times$ & & Patterson \& Carmeli (1992) \\
\hline
\end{tabular}


Table 2. Existence and effects of biogenic agents isolated from Chrysophyceae and Dinophyceae (microalgae)

\begin{tabular}{|c|c|c|c|c|c|c|}
\hline \multirow[t]{2}{*}{ Species } & \multirow[t]{2}{*}{ Biogenic agent } & \multirow[b]{2}{*}{$\begin{array}{c}\text { Anti- } \\
\text { bacterial }\end{array}$} & \multicolumn{2}{|c|}{ Effects } & \multirow[b]{2}{*}{$\begin{array}{c}\text { Anti- } \\
\text { protozoan }\end{array}$} & \multirow[t]{2}{*}{ Source } \\
\hline & & & $\begin{array}{l}\text { Anti- } \\
\text { algal }\end{array}$ & $\begin{array}{l}\text { Anti- } \\
\text { fungal }\end{array}$ & & \\
\hline \multicolumn{7}{|l|}{ Chrysophyceae } \\
\hline Ochromonas dancia & Fatty acids? & & $x$ & & & Aaronson \& Bensky $(1967)$ \\
\hline Prymnesium parvum & Glycolipid/lipoprotein & & & & $\times$ & Paster (1973), Shilo (1979) \\
\hline \multicolumn{7}{|l|}{ Dinophyceae } \\
\hline Protogonyaulax tamarensis & Terpenes/carbohydrates & $x$ & & & & Burkholder et al (1960) \\
\hline Prorocentrum micans & Terpenes/carbohydrates & $\times$ & & & & Burkholder et al. (1960) \\
\hline Goniodoma sp. & Goniodomin & $\times$ & & & & Sharma et al. (1968) \\
\hline Peridinium bipes & Fatty acids, chlorophyll $c$ & $x$ & $x$ & & & Uchida et al. (1988) \\
\hline Prorocentrum lima & Polyether compounds & & & $x$ & & Nagai et al. (1990) \\
\hline Dinophysis fortii & Polyether compounds & & & $x$ & & Nagai et al. (1990) \\
\hline Gambierdiscus toxicus & Polyether compounds & & & $x$ & & Nagai et al. (1990) \\
\hline
\end{tabular}

\section{Biogeñic agenis isoỉated from marine invertebrates}

In recent years several comprehensive accounts of antifungal, antibacterial, antialgal, antiviral and pharmacological activities of biogenic subsiances isolated from several species of marine invertebrates have been published (Targett et al. 1983, Bakus \& Kawaguchi 1984, Wahl 1987, Sears et al. 1990, Ireland et al. 1993). From 1977 to 1987,1595 bioactive compounds were isolated from marine invertebrates worldwide, predominantly sponges, coelenterates, bryozoans and ascidians (Ireland et al. 1993). Tests for medical purposes were performed, for instance, with bryostatines from bryozoans with antitumoral properties, with didemnines from ascidians with antiviral and antitumoral activities and palytoxin from corals with neurotoxic effects (Ireland et al. 1993). Furthermore, a variety of sessile marine invertebrates contain secondary metabolites affecting the settlement of fouling organisms. It is known that representatives of Porifera, Cnidaria and Tunicata are rarely overgrown by epi- phytic organisms. They frequently produce high concentrations of biogenic agents with potent antifoulant activities. Especially octocorals and sponges are a rich source of compounds that act as antifoulants (Willemsen \& Ferrari 1993).

Targett et al. (1983) determined that Leptogorgia virgulata and $L$. setacea contain high concentrations of homarine, which inhibited growth of the biofouling diatom Navicula salinicola. Extracts of $L$. virgulata and Neosimnia uniplicata, a snail of $L$. virgulata, strongly inhibited the settlement of the barnacle Balanus amphitrite. Bioassay-directed purification of L. virgulata extracts led to the identification of 2 diterpenoid hydrocarbons, pukalide and epoxypukalide, as antifouling agents (Gerhart et al. 1988). Many investigations with new species of octocorals and sponges result in the discovery of new compounds, e.g. herbacin, a new furanosequiterpene from the marine sponge Dysidea herbacea (Sarma et al. 1986).

A series of chemical compounds, like lactons, fatty acids, bromopyrroles, homarine, herbacin, pukalides,

Table 3. Existence and effects of biogenic agents isolated from Chlorophyceae (microalgae)

\begin{tabular}{|c|c|c|c|c|}
\hline \multirow[t]{2}{*}{ Species } & \multirow[t]{2}{*}{ Biogenic agent } & \multicolumn{2}{|c|}{ Effects } & \multirow[t]{2}{*}{ Source } \\
\hline & & Antibacterial & Antialgal & \\
\hline Stichococcus mirabilis & Fatty acids? & $x$ & & Harder \& Oppermann (1953) \\
\hline Protosiphon botryoides & Fatty acids? & $\times$ & & Harder \& Oppermann (1953) \\
\hline Chlamydomonas reinhardii & Fatty acids & & $x$ & Proctor $(1956)$ \\
\hline Hydrodictyum reticulatum & Fatty acids & $x$ & & Olfers-Weber \& Mihm (1978) \\
\hline Dictyosphaerium pulchellum & Methanolic extracts & $x$ & & Cannell et al. (1988) \\
\hline Klebsormidium crenulatum & Methanolic extracts & $\times$ & & Cannell et al. (1.988) \\
\hline Chlorokybus atmophyticus & Acetone extracts & $x$ & & Cannell et al. (1988) \\
\hline Pleurastrum terrestre & Methanolic extracts & $x$ & & Cannell et al. (1.988) \\
\hline Staurastrum gracile & Methanolic extracts & $x$ & & Cannell et al. (1988) \\
\hline Chlorococcum sp. & Aqueous extract & $x$ & & Ohta et al. (1993) \\
\hline Chlorococcum HS-101 & $\alpha$-Linolenic acid & $x$ & & Ohta et al. (1993) \\
\hline
\end{tabular}


Table 4. Existence and effects of biogenic agents isolated from Phaeophyceae (macroalgae). S.: Spirorbis; M.: Mytilus

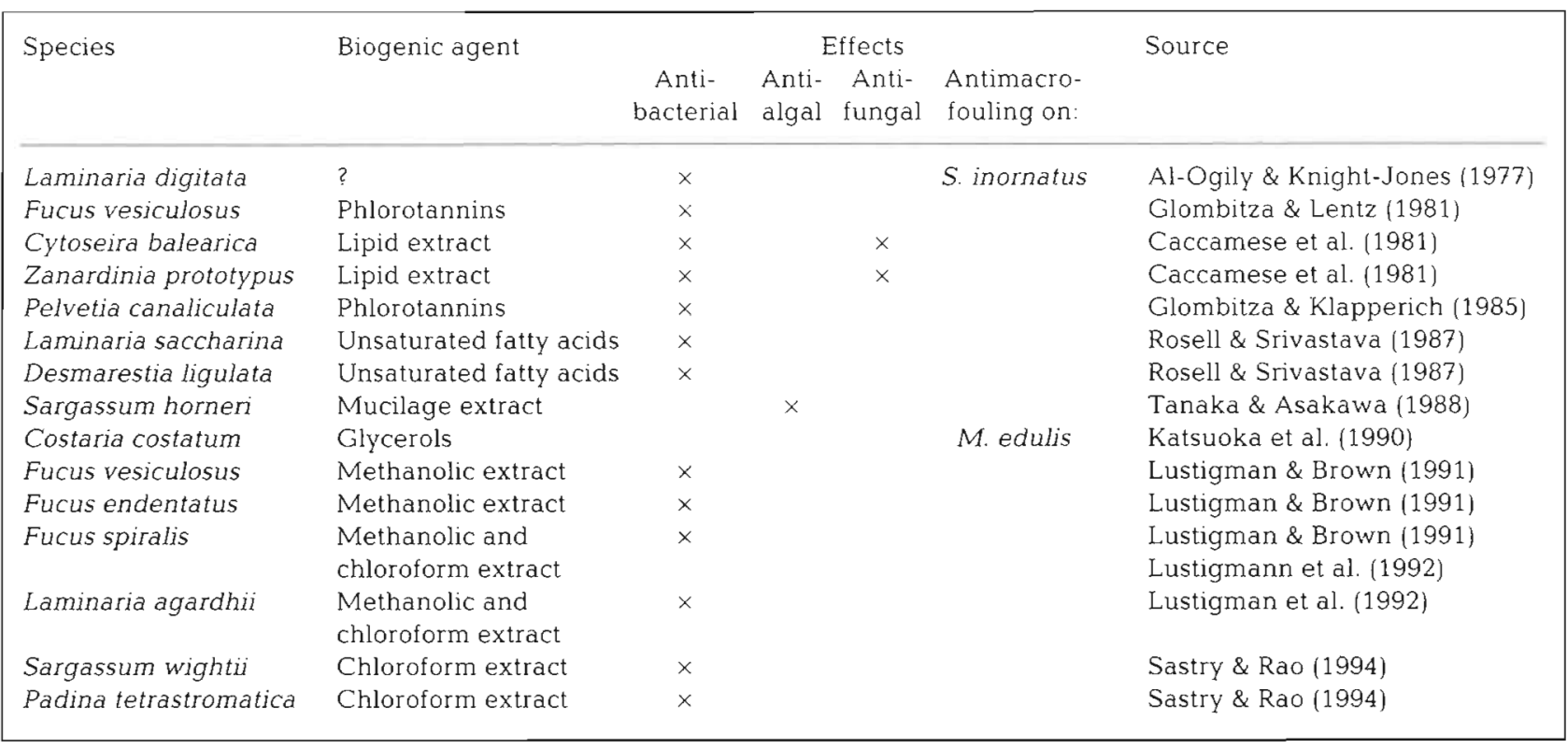

peptides, steroids and saponins, isolated from several species of Porifera, Cnidaria, Tunicata and Mollusca with antibacterial, antialgal activities and activities preventing the settlement by macrofouling organisms are listed in Table 6. In some cases, the structure of the active agent has not yet been clarified (Standing et al. 1984, Ware 1984, Rittschof et al. 1985, Sears et al. 1990, Mary et al. 1991) (Table 6). Even the results of tests of 51 sponge extracts under field conditions for their ability to prevent biofouling showed the potential antifouling activity of most of the tested crude extracts
(Willemsen \& Ferrari 1983) containing compounds of unknown structure. It can be seen from Table 6 that antibacterial and antialgal effects (effects against microfouling) are predominantly observed for homarine, fatty acids, peptides and steroids from Polysyncraton lacazei (ascidian) and Leptogorgia virgulata (coral) (Targett et al. 1983, Wahl 1987).

Potent antimacrofouling activity against the blue mussel Mytilus edulis and the barnacle Balanus amphitrite and other macrofouling organisms was found in extracts of several sponges (Lissodendoryx

Table 5. Existence and effects of biogenic agents isolated from Rhodophyceae, Chlorophyceae, Conjugatophyceae and Charophyceae (macroalgae). M.: Mytilus

\begin{tabular}{|c|c|c|c|c|c|c|}
\hline \multirow[t]{2}{*}{ Species } & \multirow[t]{2}{*}{ Biogenic agent } & \multirow[b]{2}{*}{$\begin{array}{c}\text { Anti- } \\
\text { bacterial }\end{array}$} & \multirow[b]{2}{*}{$\begin{array}{l}\text { Anti- } \\
\text { algal }\end{array}$} & \multicolumn{2}{|c|}{ Effects } & \multirow{2}{*}{ Source } \\
\hline & & & & $\begin{array}{l}\text { Anti- Anti- } \\
\text { fungal protozoan }\end{array}$ & $\begin{array}{l}\text { Antimacro- } \\
\text { fouling on: }\end{array}$ & \\
\hline \multicolumn{7}{|l|}{ Rhodophyceae } \\
\hline Laurencia obtusa & Laurencienyne & $x$ & & & & Caccamese et al. (1981) \\
\hline Centroceras clavulatum & Lipid extract & $x$ & & $x$ & & Caccamese et al. (1981) \\
\hline Sphaerococcus coronopifolius & Lipid extract & $x$ & & $x$ & & Caccamese et al. (1981) \\
\hline Gracilaria corticata & Chioroform extract & $x$ & & & & Sastry \& Rao (1994) \\
\hline Acanthophora delilei & Chloroform extract & $x$ & & & & Sastry \& Rao (1994) \\
\hline \multicolumn{7}{|l|}{ Chlorophyceae } \\
\hline Codium coralloides & Lipid extract & $x$ & & $x$ & & Caccamese et al. (1981) \\
\hline Caulerpa ashmeadii & Terpenoids & $x$ & & & & Paul et al. (1987) \\
\hline Undaria pinnatifida & Glycerols & & & & M. edulis & Katsuoka et al. (1990) \\
\hline Enteromorpha linza & Methanolic extract & $x$ & & & & Lustigman \& Brown (1991) \\
\hline \multicolumn{7}{|l|}{ Conjugatophyceae } \\
\hline Spirogyra sp. & Tannin? & & & $x$ & & Misra \& Sinha (1979) \\
\hline \multicolumn{7}{|l|}{ Charophyceae } \\
\hline Chara globularis & Dithiolan, trithian & & $x$ & & & Wium-Andersen et al. (1982) \\
\hline
\end{tabular}


Table 6 . Existence and effects of antifouling agents isolated from special species of marine invertebrates. Species: Agaricia lamarcki, Balanus amphitrite, Bugula neritina, Hippoporina americana, Hydroides norvegica, Laomedia bistriata, Mytilus edulis, Serpula vermicularis

\begin{tabular}{|c|c|c|c|c|c|}
\hline \multirow[t]{2}{*}{ Species } & \multirow[t]{2}{*}{ Biogenic agent } & \multirow[b]{2}{*}{$\begin{array}{l}\text { Anti- } \\
\text { bacterial }\end{array}$} & \multicolumn{2}{|c|}{ Effects } & \multirow[t]{2}{*}{ Source } \\
\hline & & & $\begin{array}{l}\text { Anti- } \\
\text { algal }\end{array}$ & $\begin{array}{l}\text { Antimacro- } \\
\text { fouling on: }\end{array}$ & \\
\hline \multicolumn{6}{|l|}{ Porifera } \\
\hline Xestospongia halichondriodes & $?$ & & & B. neritina & Ware (1984) \\
\hline $\begin{array}{l}\text { Placortis } \\
\text { halichondroides }\end{array}$ & $\begin{array}{l}\text { Lactons, phenols, } \\
\text { cyclic peroxides }\end{array}$ & & & A. lamarcki & Porter \& Targett (1988) \\
\hline Lissodendoryx isodictyalis & Terpenoids? & & & B. amphitrite & Sears et al. (1990) \\
\hline Agelas conifera & Bromopyrroles & & & B. amphitrite & Keifer et al.(1991) \\
\hline Dysidea herbacea & $\begin{array}{l}\text { Herbicin } \\
\text { (furanosesqui- } \\
\text { terpene) }\end{array}$ & & & $\begin{array}{l}\text { L. bistriata } \\
\text { H. americana } \\
\text { H. norvegica } \\
\text { S. vermicularis }\end{array}$ & Sarma et al. (1991) \\
\hline Phyllospongia papyracea & Fatty acids & & & $\begin{array}{l}\text { M. edulis } \\
\text { B. amphitrite }\end{array}$ & Goto et al. (1992) \\
\hline \multicolumn{6}{|l|}{ Cnidaria (Anthozoa) } \\
\hline Leptogorgia setacea & Homarine & & $x$ & & Targett et al. (1983) \\
\hline L. virgulata & Homarine & & $x$ & & Targett et al. (1983) \\
\hline L. virgulata & $?$ & & & B. amphitrite & Standing et al. (1984) \\
\hline L. virgulata & $?$ & & & B. amphitrite & Rittschof et al. (1985) \\
\hline Muricea fruticosa & Muricins (saponis) & & $x$ & & Bandurraga \& Fenical (1985) \\
\hline Renilla reniformis & Diterpenes & & & B. amphitrite & Keifer et al. (1986) \\
\hline Renilla reniformis & $?$ & & & B. amphitrite & Rittschof et al. (1986) \\
\hline L. virgulata & $\begin{array}{l}\text { Pukalide, epoxypukalid } \\
\text { (diterpenoids) }\end{array}$ & & & B. amphitrite & Gerhart et al. (1988) \\
\hline Suberogorgia suberosa & $?$ & & & B. amphitrite & Mary et al. (1991) \\
\hline Spongodes sp. & $?$ & & & B. amphitrite & Mary et al. (1991) \\
\hline Solenocaulon tortuosum & ? & & & B. amphitrite & Mary et al. (1991) \\
\hline Echinogorgia complexa & $?$ & & & B. amphitrite & Mary et al. (1991) \\
\hline Juncella juncea & $?$ & & & B. amphitrite & Mary et al. (1991) \\
\hline \multicolumn{6}{|l|}{ Mollusca (Gastropoda) } \\
\hline Neosimnia uniplicata & $?$ & & & B. amphitnte & Gerhart et al.(1988) \\
\hline \multicolumn{6}{|l|}{ Chordata (Ascidiacea) } \\
\hline Polysyncraton lacazei & $\begin{array}{l}\text { Fatty acids, peptides, } \\
\text { steroids }\end{array}$ & $x$ & $x$ & $\begin{array}{l}\text { Eggs of } \\
\text { sea urchins }\end{array}$ & Wahl (1987) \\
\hline
\end{tabular}

isodictyalis, Phyllospongia papyracea, Agelas conifera, Dysidea herbacea) and corals (e.g. Leptogorgia virgulata, L. setacea, Renilla reniformis, Suberogorgia suberosa, S. tortuosum and others) (Table 6).

\section{LITERATURE CITED}

Aaronson S, Bensky B (1967) Effect of aging of a cell population on lipids and drug resistance in Ochromonas danica. J Protozool 14:76-78

Al-Ogily SM, Knight-Jones EW (1977) Antifouling role of antibiotics produced by marine algae and bryozoans Nature 265:728-729

Baghi SN, Palod A, Chauhan VS (1990) Algicidal properties of a bloom-forming blue-green alga, Oscillatoria spec. $\mathrm{J}$ basic Microbiol 30:21-29

Baier RE (1984) Initial events in microbial film formation. In: Costlow JD, Tipper RC (eds) Marine biodeterioration: an interdisciplinary study. E \& FN Spon Ltd, London, p 57-62
Bakus GJ, Kawaguchi M (1984) Toxins from marine organisms: studies on antifouling. In: Bolis L, Zadunaisky I, Gilles $R$ (eds) Toxins, drugs and pollutants in marine animals. Springer-Verlag, Berlin, p 43-46

Bandurraga MM, Fenical W (1985) Isolation of the muricins evidence of a chemical adaption against fouling in the marine octocoral Muricea fruticosa (gorgonacea). Tetrahedron 41:1057-1065

Bloor S, England RR (1991) Elucidation and optimization of the medium constituents controlling antibiotic production by the cyanobacterium Nostoc muscorum. Enzyme microb Tech 13:76-81

Bonjouklian R, Smitka TA, Doolin LE, Molloy RM, Debono M, Shaffer SA (1991) Tjipanazoles, new antifungal agents from the blue-green alga Tolypothrix tjipanasensis. Tetrahedron 47:7739-7750

Booth WE (1981) A method for removal of some epiphytic diatoms. Botanica mar 24:603-609

Branscomb ES, Rittschof D (1984) An investigation of low frequency sound waves as a means of inhibiting barnacle settlement. J exp mar Biol Ecol 79:149-154 
Burkholder PR, Burkholder LM, Almodovar LR (1960) Antibiotic activity of some marine algae of Puerto Rico. Botanica mar 2:149-156

Caccamese S, Azzolina R, Furnari G, Cormaci M, Grasso S (1981) Antimicrobial and antiviral activities of some marine algae from Eastern Sicily. Botanica mar 14:365-367

Cannell RJP, Owsianka AM, Walker JM (1988) Results of a large-scale screening programme to detect antibacterial activity from freshwater algae. Br phycol J 23:41-44

Cardllina JH II, Moore RE, Arnold EV, Clardy J (1979) Structure and absolute configuration of malyngolide, an antibiotic from the marine blue-green alga Lyngbya majuscula Gomont. J org Chem 44:4039-4042

Caron DA, Sieburth JMcN (1981) Disruption of the primary fouling sequence on fiber glass-reinforced plastic submerged in the marine environment. Appl environ Microbiol 41:268-273

Characklis WG (1981) Fouling biofilm development: a process analysis. Biotech Bioengin 23:1923-1960

Chet I, Asketh P, Mitchell R (1975) Repulsion of bacteria from marine surfaces. Appl Microbiol 30:1043-1045

Chet I, Mitchell R (1976) Control of marine fouling by chemical repellents. In: Sharpley VM, Kaplan AM (eds) Proc 3rd Int Biodegradation Symp. Applied Science, London, p 515-521

Christie AO, Dalley R (1987) Barnacle fouling and its prevention. In: Southward AJ (ed) Barnacle biology. AA Balkema, Rotterdam, p 419-433

Christie AO, Evans LV, Shaw M (1970) Studies of the shipfouling algae Enteromorpha II. The effect of certain enzymes on the adhesion of zoospores. Ann Bot 34 : $467-482$

Cinti G, Gombach ML, Renni L (1987) Slime inhibiting antifouling paints. Polymers Paint Colour J 177:66-68

Colwell RR (1983) Biotechnology in the marine sciences. Science 222:19-24

Cooksey B, Cooksey KE (1980) Calcium is necessary for motility in the diatom Amphora coffeaeformis. PI Physiol 65 $129-131$

Cooksey B, Cooksey KE, Millar CA, Paul JH, Rubin R, Webster $D$ (1984) The attachment of microfouling diatoms. In: Costlow JD, Tipper RC (eds) Marine biodeterioration: an interdisciplinary study. E \& FN Spon Ltd, London, p $167-172$

Cooksey KE (1981) Requirement for calcium in adhesion of a fouling diatom to glass. Appl environ Microbiol 41: $1378-1383$

Corfield AP, Schauer R (1982) Occurrence of sialic acid. Cell Biol Monogr 10:5-50

Costerton JW, Geesey GG, Cheng KJ (1978) How bacteria stick. Scient Am 238:86-95

Cuba TR, Blake NJ (1983) The initial development of a marine fouling assemblage on a natural substrate in a subtropical estuary. Botanica mar 26:259-264

Dalley R (1987) Legislation affecting tributyltin antifoulings J Coatings Technol 59:113-119

Daniel GF, Chamberlain AHL, Jones EBG (1987) Cytochemical and electron microscopical observations on the adhesive materials of marine fouling diatoms. Br phycol J 22:101-118

Dempsey MJ (1981) Marine bacterial fouling: a scanning electron microscope study. Mar Biol 61:305-315

De Mule MCZ, De Caire GZ, De Cano MS, De Halperin DR (1991) Bioactive compounds from Nostoc muscorum (cyanobacteria). Cytobios 66:169-172

Dexter SC (1978) Influence of substratum critical surface tension on bacterial adhesion - in situ studies. J coll Interf
Sci $70: 346-354$

Dhar HP, Bockris JOM, Lewis DH (1981) Electrochemical inactivation of marine bacteria. J Electrochem Soc 128 : $229-231$

Eng-Wilmot DL, McCoy LF, Martin DF (1979) Isolation and synergism of a red tide (Gymnodinium Breve) cytolic factor(s) from cultures of Gomphosphaeria aponina. In: Taylor DL, Seliger HH (eds) Toxic dinoflagellate blooms. Elsevier, Amsterdam, p 355-360

Evans LV (1981) Marine algae and fouling: a review with particular reference to shipfouling. Botanica mar 24:167-171

Ferreira S, Seeliger M (1985) The colonization process of algal epiphytes on Ruppia maritime L. Botanica mar 28:245-249

Fletcher $M$ (1979) The attachment of bacteria to surfaces in aquatic environments. In: Ellwood DC, Melling J, Rutter P (eds) Adhesion of microorganisms to surfaces. Academic Press, Inc, London, p 87-108

Fletcher M. Floodgate GD (1973) An electron-microscopic demonstration of an acidic polysaccharide involved in the adhesion of a marine bacterium to solid surfaces. J gen Microbiol 74:325-334

Fletcher M, Loeb GI (1979) Influence of substratum characteristics on the attachment of a marine pseudomonad to solid surfaces. Appl environ Microbiol 37:67-72

Fletcher RL, Baier RE (1984) Influence of surface energy on the development of the green alga Enteromorpha. Mar Biol Lett 5:251-254

Flores E, Wolk CP (1986) Production, by filamentous, nitrogen-fixing cyanobacteria, of a bacteriocin and of other antibiotics that kill related strains. Arch Microbiol 145 : $215-219$

Gerencser VF, Barnothy MF, Barnothy JM (1962) Inhibition of bacterial growth by magnetic fields. Nature 196:539-541

Gerhart DJ, Rittschof D, Mayo SW (1988) Chemical ecology and the search for marine antifoulants: studies of a predator-prey symbiosis. J chem Ecol 14:1903-1915

Gitlitz MH (1981) Recent developments in marine antifouling coatings. J Coatings Technol 53:46-52

Gleason FK, Baxa CA (1986) Activity of the natural algicide, cyanobacterin, on eukaryotic microorganisms. FEMS Microbiol Lett 33:85-88

Gleason FK, Paulson JL (1984) Site of action of the natural algicide, cyanobacterin, in the blue-green alga, Synechococcus sp. Arch Microbiol 138:273-277

Glombitza KW, Klapperich K (1985) Antibiotics from algae XXXIV Cleavage of the high-molecular-weight methylated phlorotannin fraction from the brown alga Pelvetia canaliculata. Botanica mar 28:139-144

Glombitza KW, Lentz G (1981) Antibiotics from algae. XXVIIl. Cleavage of high molecular phlorotannin derivatives from the brown alga Fucus vesiculosus L. Tetrahedron 37 : 3861-3866

Golchert HJ (1993) Ökonomische Notwendigkeit für den Einsatz von Antifoulingbeschichtungen auf seegehenden Schiffen. In: Watermann B (ed) Antifouling im Meer Gefahren durch Schiffsanstriche. SDN Kolloquium, Schriftenreihe der Schutzgemeinschaft Deutsche Nordseeküste eV, Wilhelmshaven, p 14-39

Goto R, Kado R, Muramoto K, Kamiya H (1992) Fatty acids as antifoulants in a marine sponge. Biofouling 6:61-68

Grant GT, Morris ER, Rees DA, Smith PJC, Thom D (1973). Biological interactions between polysaccharides and divalent cations: the eggbox model. FEBS Lett 32:195-198

Gromov BV, Vepritskiy AA, Titova NN, Mamkayeva KA, Alexandrova OV (1991) Production of the antibiotic cyanobacterin LU-1 by Nostoc linckia CALU 892 (cyanobacterium). J appl Phycol 3:55-59 
Gross EM, Wolk CP, Jütner F (1991) Fischerellin, a new allelochemical from the freshwater cyanobacterium Fischerella muscicola. J Phycol 27:686-692

Harder R, Oppermann A (1953) Über antibiotische Stoffe bei den Grünalgen Stichococcus bacillaris und Protosiphon botryoides. Arch Microbiol 19:398-401

Haug A (1976) The influence of borate and calcium on the gel formation of a sulfated polysaccharide from Ulva lactuca. Arch Chem Scand B 30:562-566

Humphrey BA, Dickson MR, Marshall KC (1979) Physicochemical and in situ observations on the adhesion of gliding bacteria to surfaces. Arch Microbiol 120:231-238

Humphries M, Jaworzyn JF, Cantwell JB (1986) The effect of a range of biological polymers and synthetic surfactants on the adhesion of a marine Pseudomonas spec. strain NCMB 2021 to hydrophilic and hydrophobic surfaces. FEMS Microbiol Ecol 38:299-308

Imam SH, Bard RF, Tosteson TR (1984) Specificity of marine microbial surface interactions. Appl environ Microbiol 48:833-839

Ireland CM, Copp BR, Foster MP, MCDonald LA, Radisky DC, Swersey JC (1993) Biomedical potential of marine natural products. In: Attaway $\bar{D} H$, Zaborsky OR (eds) Marine biotechnology, Vol 1, Pharmaceutical and bioactive natural products. Plenum Press, New York, p 1-43

Ishibashi M, Moore RE, Patterson GML, Xu C, Clardy J (1986) Scytophycins, cytotoxic and antimycotic agents from the cyanophyte Scytoneurd pseudohotmann. J org Chem 51: $5300-5306$

Katsuoka M, Ogura C, Etoh H, Sakata K (1990) Galactosyland sulfoquinovosyldiacylglycerols isolated from the brown algae, Undaria pinnatifida and Costaria costata as repellents of the blue mussel, Mytilus edulis. Agric Biol Chem 54:3043-3044

Keifer PA, Rinehart KL, Hooper IR (1986) Renillafoulins, antifouling diterpenes from the sea pansy Renilla reniformis (Octocorallia). J org Chem 51:4450-4454

Keifer PA, Schwartz RE, Koker MES, Hughes RG Jr, Rittschof D, Rinehart KL (1991) Bioactive bromopyrrole metabolites from the Caribbean sponge Agelas conifera. J org Chem $56: 3319-3321$

Kühl H, Neumann H (1969) Versuche über Bewuchsschutz durch elektrische Strome. Schiff Hafen 21:562

Lewin $R$ (1984) Microbial adhesion is a sticky problem. Science 224:375-377

Little BJ (1984) Succession in microfouling. In: Costlow JD Tipper RC (eds) Marine biodeterioration: an interdisciplinary study. E \& FN Spon Ltd, London, p 63-67

Loeb GJ, Neihof RA (1975) Marine conditioning films. Adv Chem Ser 145:319-335

Lustigman B, Brown C (1991) Antibiotic production by marine algae isolated from the New York/New Jersey Coast. Bull environ Contam Toxicol 46:329-335

Lustigman B, Lee LH, Thees N, Masucci J (1992) Production of antibacterial substances by macroalgae of the New York/New Jersey Coast, USA. Bull environ Contam Toxicol 49:743-749

Maki JS, Rittschof D, Costlow JD, Mitchell R (1988) Inhibition of attachment of larval barnacles, Balanus amphitrite, by bacterial films. Mar Biol 97:199-206

Marshall KC (1980) Microorganisms and interfaces. BioSci 30:246-248

Marshall KC, Stout R, Mitchell R (1971) Mechanisms of the initial events in the sorption of marine bacteria to surfaces. $J$ gen Microbiol 68:337-348

Mary V, Mary A, Rittschof D, Sarojini R, Nagabhushanam R (1991) Compounds from octocorals that inhibit barnacle settlement: isolation and biological potency. In: Thompson MF, Sarojini R, Nagabhushanam R (eds) Bioactive compounds from marine organisms with emphasis on the Indian Ocean, Indo-United States Symp. AA Balkema, Rotterdam, p 333-339

Mason CP, Edwards KR, Carlson RE, Pignatello J, Gleason FK, Wood JM (1982) Isolation of chlorine-containing antibiotic from the freshwater cyanobacterium Scytonema hofmanni. Science 215:400-402

Misra A, Sinha R (1979) Algae as drug plants in India. In: Hoppe HA, Levring $T$, Tanaka $Y$ (eds) Marine algae in pharmaceutical science, Vol 1. De Gruyter, Berlin, p $237-242$

Mitchell R, Kirchman D (1984) The microbial ecology of marine surfaces. In: Costlow JD, Tipper RC (eds) Marine biodeterioration: an interdisciplinary study. E \& F N Spon Ltd, London, p 49-58

Moore RE, Yang XG, Patterson GML (1987) Fontonamide and anhydrohapaloxindole $A$, two new alkaloids from the blue-green alga Hapalosiphon fontinalis. J org Chem 52 3773-3777

Morse DE (1984) Biochemical control of larvài recruitment and marine fouling. In; Costlow JD, Tipper RC (eds) Marine biodeterioration: an interdisciplinary study. E \& FN Spon Ltd, London, p 49-58

Murakami N, Morimoto T, Imamura H, Ueda T, Nagai SI. Sakakibara J. Yamada N (1991) Studies on glyculipids. 3. Glyceroglycolipids from an axenically cultured cyanobacterium Phormidium tenue. Chem Pharm Bull Tokyo 39 $2277-2281$

Nagai H, Satake M, Yasumoto T (1990) Antimicrobial activities of polyether compounds of dinoflagellate origins. J appl Phycol 2:305-308

Oehlmann J, Stroben E, Bettin C, Fioroni P (1993) TBTinduzierter Imposex und seine physiologischen Ursachen bei marinen Vorderkiemenschnecken. In: Watermann B (ed) Antifouling im Meer-Gefahren durch Schiffsanstriche. SDN Kolloquium, Schriftenreihe der Schutzgemeinschaft Deutsche Nordseeküste eV, Wilhelmshaven, p 58-72

Ohta S, Chang T, Ikegami N, Kondo M, Miyata $H$ (1993) Antjbiotic substance produced by a newly isolated marine microalga, Chlorococcum HS-101 Bull environ Contam Toxicol 50:171-178

Olfers-Weber R, Mihm U (1978) Untersuchungen zur ökologischen Bedeutung der Massenentwicklung von Hydrodictyon reticulatum in Trinkwasserinfiltrationsbecken III. Identifizierung der Wirkstoffe durch spektroskopische Methoden in Verbindung mit Gaschromatographie. Zbl Bakteriol Parasitenk, Erste Abt Orig, Reihe B 169: $287-294$

Paster Z (1973) Pharmacology and mode of action of prymnesin. In: Martin DF, Padilla GM (eds) Marine pharmacognosy: action of marine biotoxins at the cellular level. Academic Press, New York, London

Patterson GML, Carmeli S (1992) Biological effects of tolytoxin (6-hydroxy-7-0-methylscytophycin b), a potent bioactive metabolite from cyanobacteria. Arch Microbiol 157: $406-410$

Paul JH, Jeffrey WH (1985) Evidence for separate adhesion mechanisms for hydrophilic and hydrophobic surfaces in Vibrio proteolytica. Appl environ Microbiol 50:431-437

Paul VJ, Littler MM, Littler DS, Fenical W (1987) Evidence for chemical defense in tropical green alga Caulerpa ashmeadii (Caulerpaceae: Chlorophyta): isolation of new bioactive sesquiterpenoids. J chem Ecol 13:1171-1185

Pedersen M. Da Silva EJ (1973) Simple brominated phenols 
in the blue-green alga Calothrix brevissima West. Planta 115:83-86

Percival E (1979) The polysaccharides of green, red and brown seaweeds: their basic structure, biosynthesis and function. Br phycol J 14:103-117

Peterson SM, Batley GE, Scammell MS (1993) Tetracycline in antifouling paints. Mar Pollut Bull 20:96-100

Porter D. Targett NM (1988) Allelochemical interactions between sponges and corals. Biol Bull 175:230-239

Price RR, Patchan M, Clare A, Rittschof D, Bonaventura J (1992) Performance enhancement of natural antifouling compounds and their analogs through microencapsulation and controlled release. Biofouling 6:207-216

Proctor VW (1956) Studies of algal antibiosis using Haematococcus and Chlamydomonas. Limnol Oceanogr 1:125-139

Reuter W, Köttgen E, Bauer C, Gikok W (1982) Biological significance of sialic acid. Cell Biol Monogr 10:263-305

Rittschof D, Hooper IR, Branscomb ES, Costlow JD (1985) Inhibition of barnacle settlement and behavior by natural products from whip corals, Leptogorgia virgulata (Lamarck, 1815). J chem Ecol 11:551-563

Rittschof D, Hooper IR, Costlow JD (1986) Barnacle settlement inhibitors from sea pansies, Renilla reniformis. Bull mar Sci 39:376-382

Rittschof D, Schmidt AR, Hooper IR, Gerhart DJ, Gunster D, Bonaventura J (1991) Molecular mediation of settlement of selected invertebrate larvae. In: Thompson MF, Sarojini $R$, Nagabhushanam $R$ (eds) Bioactive compounds from marine organisms with emphasis on the Indian Ocean, Indo-United States Symp. AA Balkema, Rotterdam, p 317-330

Rosell KG, Srivastava LM (1987) Fatty acids as antimicrobial substances in brown algae. In: Ragan MA, Bird CJ (eds) Twelfth International Seaweed Symposium. Developments in hydrobiology 41 Dr W Junk Publisher, Dordrecht, p 471-475 (reprinted from Hydrobiologia 151/152)

Sarma NS, Rambabu M, Anjaneyulu ASR, Rao CBS (1986) Structure and stereo-chemistry of herbacin, new furanosesquiterpene from the marine sponge, Dysidea herbacea. Ind J Chem 25 B:1001-1003

Sarma NS, Rao KS, Viswanadham B (1991) Settling responses and progression in community development of selected macrofouling organisms to a recently isolated sponge metabolite, herbacin, at Visakhapatnam Harbor, Bay of Bengal. In: Thompson MF, Sarojini R, Nagabhushanam R (eds) Bioactive compounds from marine organisms with emphasis on the Indian Ocean, Indo-United States Symp. AA Balkema, Rotterdam, p 341-350

Sastry VMVS, Rao GRK (1994) Antibacterial substances from marine algae: successive extraction using benzene, chloroform and methanol. Botanica mar 37:357-360

Sawant SS, Gary A, Wagh AB (1992) Antifouling property of the fruits of Randia brandisij (Rubiaceae) and Sapindus trifoliatus (Sapindaceae). Ind J mar Sci 21:226-227

Sawant SS, Wagh AB (1994) Studies on the antifouling properties of some natural products from Goa. In: Thompson MF, Nagabhushanam R, Sarojini R, Fingerman $M$ (eds) Recent developments in biofouling control. Oxford \& IBH Publ Co, New Delhi, p 275-282

Schulz S, Subklev HJ (1964) Wirkungen von Ultraschall auf niedere Wasserorganismen. Biol Zentralbl 3:297-302

Sears MA, Gerhart DJ, Rittschof D (1990) Antifouling agents from marine sponge Lissodendoryx isodictyalis Carter. J chem Ecol 16:791-799

Sharma GM, Michaels L, Burkholder PR (1968) Goniodomin, a new antibiotic from a dinoflagellate. $J$ Antibiot 21: $659-664$
Shilo M (1979) The toxic principles of Prymnesium parvum In: Carmichael WW (ed) The water environment. Algal toxins and health. Plenum Press, New York, p 37-47

Sieburth JMCN, Tootle JL (1981) Seasonality of microbial fouling on Ascophyllum nodosum (L.) Lejol, Fucus vesiculosus L., Polysiphonia lanosa (L.) Tandy and Chondrus crispus Stackh. J Phycol 17:57-64

Snell TW (1980) Blue-green algae and selection in rotifer populations. Oecologia 46:343-346

Sönnichsen H (1993) Antifouling in der Natur und Perspektiven nichttoxischer Schiffsanstriche. In: Watermann B (ed) Antifouling im Meer - Gefahren durch Schiffsanstriche. SDS Kolloquium. Schriftenreihe der Schutzgemeinschaft Deutsche Nordseeküste $\mathrm{eV}$, Wilhelmshaven, $\mathrm{p}$ 93-112

Standing DJ, Hooper IR, Costlow JD (1984) Inhibition and induction of barnacle settlement by natural products present in octocorals. J chem Ecol 10:823-834

Suzuki T, Matsuda R, Saito Y (1992) Molecular species of tri-n-butyltin compounds in marine products. J Agric Food Chem 40:1437-1443

Tanaka N, Asakawa A (1988) Allelophatic effect of mucilage released from a brown alga Sargassum horneri on marine diatoms. Nippon Suisan Gakk 54:1711-1714

Targett NM, Bishop SS, Mc Conell OJ, Yoder JA (1983) Antifouling agents against the benthic marine diatom, Navicula salinicola: homarine from the gorgonians Leptogorgia virgulata and $L$. setaca and analogs. J chem Ecol 9: $817-829$

Teuscher E, Lindequist U (1988) Biogene Gifte. AkademieVerlag, Berlin

Turakhia MH, Characklis WG (1989) Activity of Pseudomonas aeruginosa in biofilms: effect of calcium. Biotechnol Bioeng 33:406-414

Uchida A, Shimada A, Ishida Y (1988) Antibacterial and antialgal substances produced by the dinoflagellate Peridinium bipes. Bull Jap Soc scient Fish 54:1941-1945

Uhler A.D, Durell GS, Steinhauer WG, Spellacy AM (1993) Tributyltin levels in bivalve mollusks from the east and west coasts of the United States: results from the 1988-1990 national status and trend mussel watch project. Environ Toxicol Chem 12:139-153

Von Oertzen JA, Scharf EM, Aindt EA, Sandrock $S$, Dettmann L, Holzapfel H, Ringstorf H, Kohn H, Günther R (1989) Spezialstudie 'Alternative Antifouling Systeme' Fachbereich Biologie, Universität Rostock

Wahl M (1987) Epibiosis und Antifouling im Meer. Die Abwehrmechanismen der kolonialen Seescheide P. lacazei, gegenüber dem Besiedlungsdruck durch potentielle Epibionten. Dissertation, Math-Nat Fakultät, Universität Kiel

Wahl M (1989) Marine epibiosis. I. Fouling and antifouling: some basic aspects. Mar Ecol Prog Ser 58:175-189

Walt DR, Smulow JB, Turesky SS, Hill RG (1985) The effect of gravity on initial microbial adhesion. J coll Interf Sci $107: 334-336$

Ware GN (1984) The patterns and mechanisms of antifouling in some temperate sponges. PhD dissertation, Duke University, Durham, NC

Wigglesworth-Cooksey B, Cooksey KE (1992) Can diatoms sense surfaces?: state of our knowledge. Biofouling 5: $227-238$

Willemsen PR, Ferrari GM (1993) The use of antifouling compounds from sponges in antifouling paints. Surf Coat int 10:423-427

Wium-Andersen S, Anthoni U, Christophersen C, Houen G (1982) Allelopathic effects on phytoplankton by sub- 
stances isolated from aquatic macrophytes (Charales) Oikos 39:187-190

Zahuranec BJ (1991) The United States Navy and research on bioactive substances from the sea. In: Thompson $\mathrm{MF}_{\text {, }}$

This review was submitted to the editor
Sarojini R, Nagabhushanam R (eds) Bioactive compounds from marine organisms with emphasis on the Indian Ocean, an Indo-United States Symposium. AA Balkema, Rotterdam, p $4-6$

Manuscript first received: September 12, 1994

Revised version accepted: February 15, 1995 

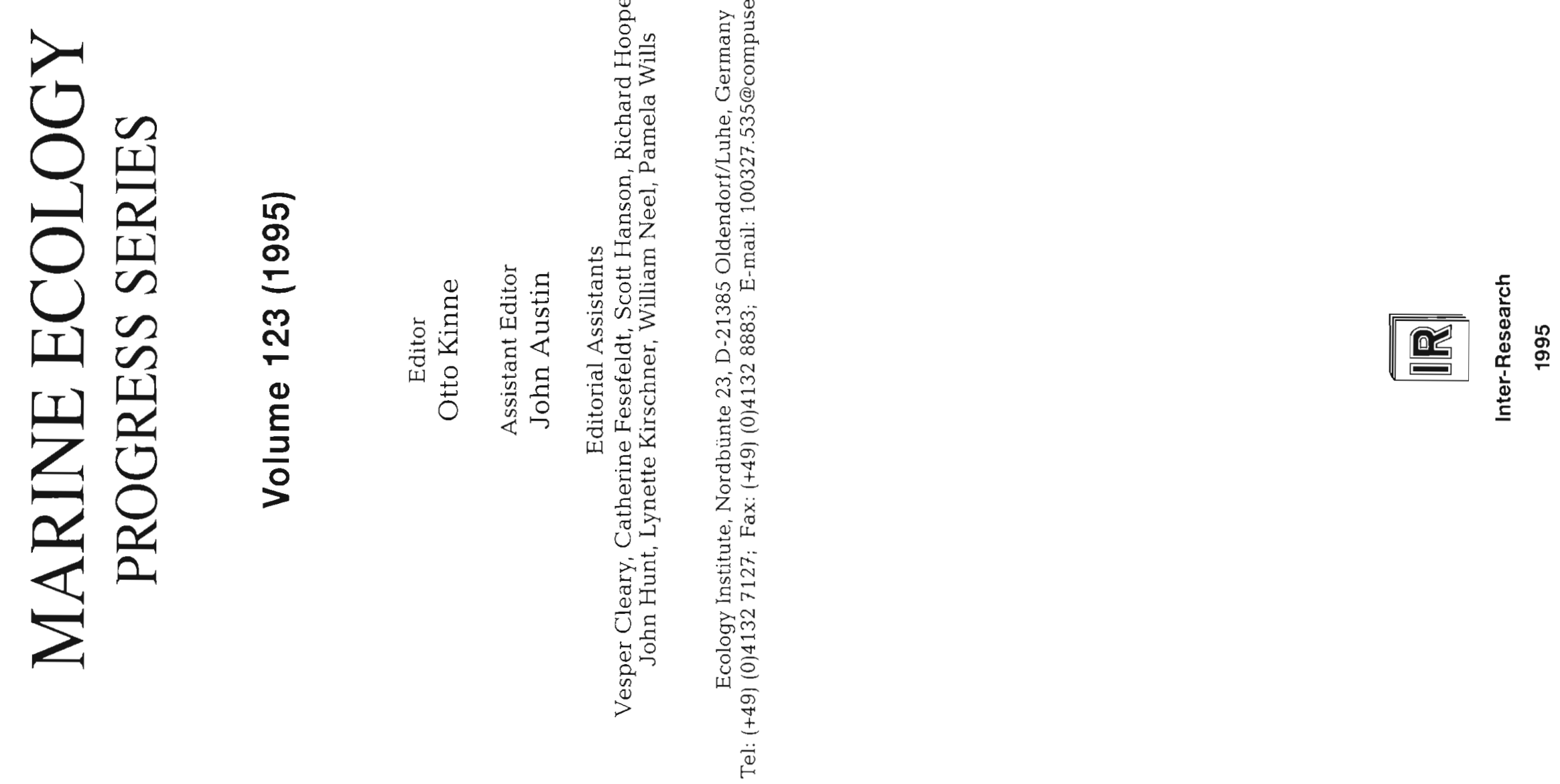


\section{Editorial Advisors}

Marine Ecology Progress Series (MEPS) is edited in cooperation with the Editorial Advisors (EAs) listed below and about 400 Anonymous Referees. EAs are appointed for a 4 -year period re-appointment is possible. They critically evaluate the scientific merits of submitted manuscripts. EAs also recommend authors and topics for reviews to be published in MEPS.

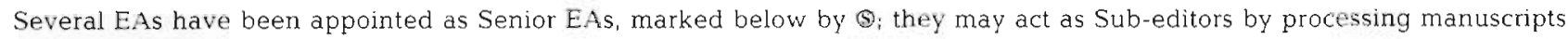
themselves, i.e. see them through a rigorous 3-referee evaluation process and present them to the Editor who reserves the right of final acceptance.

\section{ENVIRONMENTAL FACTORS}

\section{Ahmed, S. I.}

School of Oceanography, WB-10, Univ. of Washington, Seattle, WA 98195 , USA

Physiological ecology of phytoplankton; thermophilic mictoorganisms

Collos, Y. (8)

Laboratoire d'Hydrobiologie, Université de Montpellier CC 093, F-34095 Montpellier Cedex 5. France

Nitrogen metabolism of phytoplankton

Dring, M. J.

Biologische Anstalt Helgoland, D-27498 Helgoland, Germany

Physiological ecology of algae

\section{Fisher, N. S.}

Marine Sciences Research Center. State Univ, of New York, Stony Brook, NY 11794 . 5000, USA

Physiological erology of phytoplankton

Fowler, S. W. S

International Atomic Energy Agency, Marine Environment Laboratory, 19, Ave des Castellans, BP 800, MC-98012 Monaco Cedex Trace element metabolism

\section{Haedrich, R. L. (5)}

Memorial Univ, of Newfoundland, St. John's, NF, Canada A1B 3X9

Environmental effects on fishes (incl deep sea) Kasyanov, V.

Institute of Marine Biology. Academy of Sciences, Vladivostok 690032, Russia

Environmental effects on invertebrates; reproduction, development

\section{Kiørboe, T. 5}

Danish Institute for Fisheries and Marine Res., Charlottenlund Castle, DK-2920 Charlottenlund, Derımark

Physiological ecology of zooplankton, including fishes (esp. larvate)

Lawrence, J. M. (5)

Dept of Biology, Univ, of South Florida, Tampa, FL 33620, USA

Physiological ecolocyy of invertebrates

\section{Luoma, S. N.}

US Dept of the Interior, Geological Survey, Water Resources Div., 345 Middlefjeld Rd, Menlo Park, CA 94025, USA

Geochemical and biological factors affecting biochemical processes in invertebrates; contaminant effects on bicavallability

\section{McLusky, D. S. (5)}

Dept of Biological Sciences, Univ. of Stirling, Stirling FK9 4LA. Scotland

Physiological ecology of invertebrates

Meyer-Reil, L.-A.

Institut für Okologie, Schwedenhagen, D18565 Kloster/Hiddensee, Germany Environmental effects on berithic microorganisms, benthic microbial ecology

\section{Meyers, S. P. \$}

Dept of Food Science, Louisiana State Univ., Baton Rouge, LA 70803, USA

Physiological ecology of microorganisms

Paasche, E.

Dept of Biology, Univ. of Oslo, Marine Botany, POBox 1069, Blindern, N-0316 Oslo 3, Norway Physiological ecology of phytopiankton; primary production; benthic algae

Rheinheimer, G. (9)

Institut fur Meereskunde, Univ. of Kiel, Dus. ternbrooker Weg 20, D-241.05 Kiel, Ciermany Marine microbiology

\section{Riisgård, H. U. S}

Odense Univ., Biologisk Institut, Campusvej 55. DK-5230 Odense M., Denmark Environmental effects on invertebrates: bioenergetics of suspension feeders

\section{Southward, A. J.}

PIymouth Marine Laboratory, Citadel Hill, Plymouth PL1 2PB, England

Environmental effects on invertebrates

West, J. A.

School of Botany, Uniw. of Melbourne, Parkville, VIC 3052, Australia

Phvsiological ecology and reproductive biology of estuarne and manne benthic macroalgae

\section{PHYSIOLOGICAL MECHANISMS}

\section{Batty, R.S.}

Dunstaffnage Marine Research Laboratory. PO Box 3 Oban, Argy!l DA34 4AD, Scotiand Behavioural physiology of fish; feedung; predation of tish larvae

\section{Browman, H. I.}

Marine Productivity Division, MauriceLamontagne Institute, Dept of Fisheries and nceans Canada, $C P$ 1000, Miont-joili, $P Q$ Canada G5H 3Z4

Foraging behavior and development of (sensory) organs, especially in fishes.

\section{Brown, B. E.}

Dept of Biology, The University, Newcastleupon-Tyne NE1 7RU, England

Physiology and ecology of corals

Buchholz, F.

Biologische Anstalt Helgoland, Meeresstation, D-27498 Helgoland, Germany

Physiological ecology; netabolic and thermal adaptation; zooplankton; moulting

\section{Burton, R.S.}

Marine Biology Research Division 0202, Scripps Institution of Oceanography, Lniv. of California, 9500 Gilman, La Jolla, CA $92093-$ 0202, USA

Population genetics; evolutionary mechanisms in populations: biochemical and molecular genetic analyses of invertebrates

Caron, D. A. (2)

Biology Dept, Woods Hole Oceanographic Institution, Woods Hole, MA 02543, USA

Feeding, respiration and nutrient regeneraijon of protozoans

\section{Conover, R. J.}

Marine Ecology Laboratory, Bedford Institute of Oceanography, PO Box 1006, Dartmouth, NS, Canada B2Y 4 A2

Physiology and biochemistry of zooplankton

Forward, R. B., Jr

Duke Univ, Marine Laboratory Pivers Island, Beaufort, NC 28516-9721, USA

Environmental zoo-physiology; behavior; biological rhythms

\section{Gerking, S. D.}

Dent of Zoology, Arizona State Univ., Tempe, AZ 85281, USA

Fish physiology

Henley, W. J.

Dept of Botany, Oklahoma State Univ. Stillwater, OK 74078-0293, USA

Plant physiology; environmental effects on photosynthesis and respiration

\section{Holland, N. D. (8)}

Scripps Institution of Oceanography. San Diego, PO Box 1529, La Jolla، CA 92093, USA Comparative, celiular and developmental physiology
Humphrey, G. F. (S)

CSIRO Marine Biochemistry, Building $\mathrm{A} 12$ Sydney Univ., NSW 2006, Australia Photosynthesis; plankton biochemistry

Leftley, J. $W$.

Dunstaffnage Marine Research Laboratory PO Box 3, Oban, Argyll PA34 4AD, Scolland Physiology and biochemistry of microalgae (laboratory and field): nitrogen metabolism

Li, W.

Bedford Institute of Oceanogranhy, PO Box 1006, Dartmouth, NS, Canada B2Y 4 A2

Flow crtometry; photosynthetic phytoplankton; relations between phytoplankton and heterotrophic bacteroplankton

McLusky, D. S. (9)

Dept of Biological Sciences, Univ. of Stirling, Stirling FK9 4LA. Scotland

Osmorequlation in estuarine invertebrates

Naylor, E.

Schoul of Ocean Sciences, Univ of Wales, Menai Bridge Gwrnedd LL59 5EY. Wales Behavioural physiology; invertebrate biology Pawlik, J.

The Univ. of North Carolina at Wilmington, Wilmington, NC 28403-3297, USA

Chemical deiense of invertebrates: antiTouling, chemical cues

Puiseux-Dao, S.

Laboratoire de Cytophysiolorra végétale et de Toxicologie cellulaire, Université de Paris VII, 2 Place Jussieu, F-75251 Paris Cedex 05, France Ecophysiology and toxicolngy of algae; cellular biology; behavior of algae

\section{Rivkin, R. B.}

Ocean Sciences Centre, Univ. of Newfoundland, St. John's, NF, Canada A1C 5S7

Phytoplankton physiology and ecology

Shick, J. M.

Dept of Zoology, Univ, of Maine, Murray Hall, Orono, ME 04469-5751, USA

Physiological energetics of invertebrates incl. metabolic calonmetry; symbioses; hyperoxia and oxidative stress; effects of UV light; sea anemone biology

Stickle, W. B.

Zoology and Physiology, Louisiand State Univ., Baton Rouge, LA 70803-1725, USA

Ecological physiology of animals; toxicology: free amino acid regulation; effects of hypoxic. temperature and salinity; sublethal responses to pollution; toletance limits; oll pollution

Videler, J. J.

Dept of Marine Biology, Uniy of Groningen, PO Box 14, 9750 AA Haren. The Netherlands Swimming dynamics of planktorl; activity pattern and reproductive strategies of fishes; energy budgets of herbivores

Webb, P. W.

Univ, of Michigan, School of Natural Resources, 430 East University, Ann Arbor, MI 48109-1115, USA

Swimming mectianics and energetics; vertebrate behavior and distribution; energy and matera! flow through animals; growth and metabolism

West, J. A.

Dept of Plant Biology, Univ, of California, 111 Genetics Plant Biology Bldg, Berkeley, CA 94720 USA

Chemistry of red algae; molecular genetics of red and green algae

wieser, $W$.

Institut fur Zoologie der Leopold-Franzens Univ. Irinsbruck. Technikerstraße 25, A-6020 Innsbruck. Austria

Physiological ecology of invertebrates 
Zhirmunsky, A. V.

Institute of Marine Biology, Academy of

Sciences, Vladivostok 690032, Russia

Mechanisms of cellular resistance and control

\section{CULTIVATION}

Lawrence, J. M. \$

Dept of Biology, Univ. of South Florida, Tampa, FL. 33620, USA

Nutrition of invertebrates

Meyers, S. P. 8

Dept of Food Science, Louisiana State Univ, Baton Rouge, LA 70803, USA

Cultivation of microorganisms and crustaceans; aquaculture; microenvironments

Pandian, T. I.

School of Biological Sciences, Madurai Kamaraj Univ, Madurai 625021 , Tomilnadu, India Cultivation of decapods and fishes; bioenergetics

\section{Rheinheimer, G. (s)}

Institut für Meereskunde, Univ. Kiel, Düstembrooker Weg 20, D-24105 Kiel, Germany

Cultivation of microorganisms

\section{DYNAMICS}

Ahmed, S. I.

School of Oceanography, WB-10, Univ. of

Washington, Seattle, WA 98195, USA

Phytoplankton ecology; greenhouse gases and global ocean flux $x_{i}$ sedimentary microbiology mangrove ecosystems

\section{Alongi, D. M.}

Coastal Processes and Resources Program, Australian Institute of Manne Science, PMB No.

3, Townsville M.C., Q 4810, Australia

Trophic dynamics and nutrient energetics of soft-bottom benthic food webs (particulariy tropical microbes and meiofauna)

Azam, F.

Scripps lnstitution of Oceanography, Univ. of California, La Jolla, CA 92093. USA

Viral and bacterial ecology; microbial biogeo* chemistry

\section{Bak, R. P. M. ()}

Nederlands Instatuut voor Onderzoek der Zee,

Postbox 59, 1790 AB Den Burg - Texel. The

Netherlands

Coral reef ecology

Banse, $\mathbf{K}$.

School of Oceanography, Box 357940 . Univ. of Washington, Seattle, WA 98195-7940, USA Biological oceanography; plankton research

Berman, $\mathrm{T}$.

Kinneret Limnological Laboratory, PO Box 345.

Tiberias 14102, Israel

Phytoplankton ecology

Bianchi, A

C.N.R.S. ER 223, Microbiologie Marine, Campus de Luminy - case 907, 70, route Léon Lachamp,

F-13288 Marseille Cedex 9. France

Marine microbiology; ecological dynamics

Birkeland, C.

Univ of Guam, Marine Laboratory, UOG Station, Mangilao, Guam 96913

Coral-rect ecology; benthic ecology

Brattegard, $\mathrm{T}$.

Universitetet a Bergen, Institutt for fiskerl- og marin-biologi, Thormohlens gt. 55, N-5008 Bergen, Norway

Distribution and ecology of malacostracan crustaceans (esp. suprabenthic/hyperbenthic species)

\section{Browman, $\mathrm{H}$.}

Marine Productivity Division, Maurice-Lamontagne Institute, Dept of Fisheries and Oceans Canada, CP 1000, Mont-Joli, PQ, Canada G5H 3 Z4

Early life history, growth and development in tishes; fish-zooplankton interactions

Bunt, J. S.

4/6 McDonald Street, Potts Point, NSW 2011, Australia

Manqrove systems; reef studies
Caron, D. A. (s)

Biology Dept, Woods Hole Oceanographic Institution, Woods Hole, MA 02543, USA

Microbial ecology; trophic relations between pianktonic protozoans and other heterotrophic and photosynthetic organisms; pelagic detrital aggregates

Collos, Y. (S

Laboratoire d'Hydrobiologie, Unuversité de Montpellier CC 093, F-34095 Montpellier Cedex 5. France

Nitrogen cycling and primary production

Conover, R. J.

Marine Ecology Laboratory, Bedford Institute of Oceanography, PO Box 1006, Dartmouth, NS Canada $\mathrm{B} 2 \mathrm{Y} 4 \mathrm{~A} 2$

Zooplankton biology

Cushing, D. $\mathrm{H}$.

Ministry of Agriculture, Fisheries and Food Fisheries Laboratory, Lowestoft. Suffolk NR33 OHT, England

Productivity of the sea; fish-population dynamics; biological oceanography

Deibel, $\mathbf{D}$

Ocean Sciences Center, Memorial Univ. of New foundland, St. John's, NF, Canada A1C 5S7 Zooplankton ecology/nutrition, gelatinous ecology/physiology

de Jonge, $\mathrm{V}$.

National Institute for Coastal and Marine Management (RlKZ), PO Box 207, 9750 AE Haren, The Netherlands

Estuarine and coastal processes; microphytobenthos and eelgrass; process and impact of resuspension; eutrophication

Dolan, J.

Observatoure des Sciences de l'Unuvers, Université Paris VI-CNRS, Station Zoologıque, BP 28, F-06230 Villefranche-sur-Mer, France

Microbial ecology, interactions of bacteria, protists and métázóa

\section{Elliott, $M$.}

Dept of Applied Biology, Institute of Estuarine and Coastal Studies, The University, Hull HU6 7RX, England

Structure and function of fish and benthic communities

Feller, R. $\boldsymbol{J}$.

Brology and Marine Science, Belle W. Baruch Institute for Marine Biology and Coasta Research, Columbia, SC 29208, USA

Trophic dynamics and energetics of crustaceans feeding and digestion of fishes; benthic ecology of meiofauna; ecological applications of immurology and serology

Fenchel, T. 8

Marine Biological Laboratory (Univ. of Copen hagen), DK-3000 Helsingor, Denmark Population biology; microbial ecology

Field, J. G.

Univ. of Cape Town, Zool. Dept, Rondebosch 7700, Republic of South Africa

Dynamics of nearshore ecosystems

\section{Floc'h, J. Y.}

Université de Bretagne Occidentale, Lab d'Ecophysiologie et de Biochimie des Algues Marines, Faculte des Sciences, 7 av. V. Le Gorgeu, BP 452, F-29275 Brest Cedex, France Ecophysiology and biochemistry of macroalgae

\section{Foster, M. S. (s)}

Moss Landing Marine Laboratories, California State Univ., PO Box 450, Moss Landing, CA 95039-0450, USA

Population and community ecology of macroalgae

Fowler, S. W. (9)

Intemational Atomic Energy Agency, Marine Environment Laboratory, 19, Ave des Castellans, BP 800, MC-98012 Monaco Cedex

Particle flux; biogeochemistry

Fuhrman, J. (s)

Marine Biology Res. Section, Dept of Biological Sciences, Univ of Southern California Los Angeles, CA 90089-0371, USA

Planktonic microbial ecology. Productivity and growth of microorganisins, especially becteria
Furness, R. W.

Dept of Zoology, The Univ. of Glasgow Glasgow G12 8QQ, Scotland

Ecology of sea birds

Furuya, K.

Faculty of Bioresources, Mie Univ., 1515 Kamihama, Tsu 514, Japan

Phytoplankton ecology: community structure, dynamics

Gage, J. D. (s)

Dunstaffnage Marine Research Laboratory, PO Box 3, Oban, Argyll PA34 4AD, Scotland Benthos of deep sea and temperate coasts Gray, J. (s)

Institutt for Marinbiologi og Limnologi, Univ Oslo, Postboks 1064, Blindern, Oslo 3, Norway Soft-sediment ecology

Haedrich, R. L. (s)

Memorial Univ. of Newfoundland, St. John's,

NF. Canada A1B $3 \times 9$

Zoogeography; natural history of fish

Hansen, $B$.

Roskilde Univ., Instıtute I, Life Sciences \& Chemistry, PO Box 250, DK-4000 Roskilde Denmark

Laboratory and in situ studies on zooplankton; secondary production (grazing, growth, ener getics, teeding behaviour

\section{Harding, G. C.}

Marine Ecology Laboratory, Bedford Institute of Oceanography, PO Box 1006, Dartmouth, NS, Canada B2Y 4 A2

Crustacean ecology (incl. deep sea)

Heck, K. L., Jr ()

Marine Environmental Sclences Consortium, Dauphin Island Sea Lab., PO Box 369-370, Dauphin Island, AL 36528, USA

Benthic ecology, emphasis on seagrass ecosystems

Holm-Hansen, $\mathrm{O}$.

Scripps Inst. of Oceanography, Univ of California, La Jolla, CA 92093-0202, USA

Polar ecology: chemical oceanography: photosynthesis

Hoppe, H.-G.

Institut für Meereskunde, Univ. Kiel, Düsternbrooker Weg 20, D-24105 Kiel, Germany

Microbiolocy, including methods: microbial plantology

Hughes, R, N. (s)

School of Animal Biology, Univ. College North Wales, Bangor, Gwynedd LL57 2UW, Wales Ecological energetics (particularly feeding)

Karlson, R. H.

School of Life and Health Sciences Ecology Program, Univ. of Delaware, Newark, DE 19716 USA

Population and community ecology of benthic invertebrates

Kiørboe, $\mathrm{T}$.

Danish Institute for Fisheries and Marine Res. Charlottenlund Castle, DK-2920 Charlottenlund, Denmark

Biological aceanography; physical and biological processes in the pelagial with emphasis on copepods and fish larvae

Klumpp, D.

Australian Institute of Marine Science, PMB No. 3. Townsville M.C., Q 4810, Australia

Trophodynamics; animal nutrition, energy and nutrient budgets; nutrient cycling; primary pro ductivity; fish biology; seagrass, coral reef, kelp bed ecosystems

\section{Kneib, R. T.}

Marine Institute, The Univ. of Georgia, Sapelo Island, GA 31327, USA

Population and community dymamics of estuarine fishes and invertebrates; growth and mortality during early life of estuanne species; evaluation of coastal wetlands

Landry, M. R. 9

Dept of Oceanography, Univ. of Hawaii, Manoa 1000 Pope Rd, Honolulu, HI 96822, USA

Food web interactions; zooplankton ecology

Lawrence, J. M. ()

Dept of Biology, Univ. of South Florida, Tampa FL 33620, USA

Reproduction of invertebrates; nutrition 
Levings, C. (s)

West Vancouver Laboratory, 4160 Marine Dnve,

West Vancouver, BC, Canada V7V $1 N 6$

Estuarine ecolorgy of macrobenthos and fish

Lewis, J. B.

Dept of Biology, McGill Univ., Stewart Biology

Bldg, 1205 Dr. Penfield, Montreal, PQ. Canada

H3A 1 B1

Coral-reef ecology; tropical marine biology

Lewis, M. R.

Dept of Oceanography, Dalhouste Univ.

Hallfax, NS, Canada B3H $4 \mathrm{~J} 1$

Biological oceanography phytoplankton physi-

ology; modelling of physical and biological processes

Lobel, P. S.

Marine Biological Laboratory, Woods Hole, MA 02543, USA

Fish ecology, behavior biogeography; coral reefs; fisheries and oceanography

Luoma, S. N.

US Dept of the Interior, Geological Survey, Water Resources Div., 345 Middlefield Rd. Menlo Park, CA 94025, USA

Feeding ecology of deposit feeders; geochemistry of trace elements in sediments

Marcus, N. H.

Dept of Oceanography, Florida State Univ., B-169, Tallahassee, FL 32306-3048, USA

Zooplankton ecology; biology and genetics of invertebrate populations; rhythms

Mauchline, J.

Scottish Marine Biolorical Ass, Dunstaffnage Marine Research Laboratory, PO Box 3, Oban, Argyll PA34 4AD, Scotland

Deep-sea ecology; radioartive pollution

Nelson, D. M.

College of Oceanography, Oregon State Univ. Occanography Admin. Bldg 104, Corvallis, OR 97331, USA

Phytoplankton ecology; nutrient cycling

Newell, S. Y. (s)

The Univ. of Georgna, Marine Institute, Sapelo Island, GA 31327, USA

Ecology of fungi and bacteria; decomposition

Nybakken, J.

Moss Landing Marine Laboratories, California

State Univ., PO Box 450, Moss Landing, CA 95039-0450, USA

Ecology of molluscs; community structure of softsediment invertebrates

Oviatt, C. A.

Graduate School of Oceanography, Univ. of Rhode Island, Narragansett, RI 02882-1197, USA Systems ecology; primary production; nutrient and carbon cycling; role of higher trophic levels; impact of pollutants on coastal systems

Pawlik, J.

The Univ. of North Carolina at Wilmington, Wilmington, NC 28403-3297, USA

Ecology and biology of invertebrate larvae; settlement; recruitment; life histories; dispersal; natural products

Peterson, C. H. (3)

Institute of Marine Science, Univ. of N Carolina at Chapel Hill, 3407 Arendell Street, Morehead City, NC 28557, USA

Marine benthic ecology; population biology

Platt, $T$.

Marine Ecology Laboratory, Bedford Institute of Oceanography, PO Box 1006. Dartmouth, NS, Canada B2Y 4 A2

Phytoplankton ecology; prmary production

Purcell, J. E. (S)

The Univ. of Maryland Systems, Center for Environmental and Estuarine Studies, PO Box 775, Cambridge, MD 21613, USA

Zooplankton ecology and behavior; trophic dynamics; prey selection. Competition for space among benthic invertebrates

Rassoulzadegan, F. (s)

Observatoire des Sciences de l'Univers, Université Paris VI-CNRS, Station Zoologique, BP 28, F-06230 Villefranche-sur-Mer. France Microbial ecology (food webs, microbial loop); pico- and nanoplankton; planktonic flagellates and ciliates; carbon flux
Reise, $K$.

Biologische Anstalt Helgoland, D-25992 List Sylt, Germany

Benthos ecology

Rivkin, R. B.

Ocean Sciences Centre, Univ of Newfoundland, St. John's, NF, Canada A1C 5 S7

Microbial ecology; polar planktonic processes; lower food web dynamics

Savidge, G.

The Queen's Unuv. of Belfast, Marine Biology Station, The Strand, Portaferry, Co. Down, Northem Ireland

Phytoplankton ecology; primary production; photosynthesis

\section{Schneider, D. C. (5)}

Newfoundland Institute for Cold Ocean Science. Memorial Univ. of Newfoundland, St. John's, NF. Canada A1B $3 X$ ?

Dynamics of large organisms; ecology of sea birds

\section{Sherr, B. \& E. (5)}

College of Oceanography, Oregon State Univ., Oceanography Admin. Bldg 104, Corvallis, OR 97331. USA

Microbial fond webs; trophic roles of phagotrophic protozoans

\section{Southward, A. J.}

Plymouth Marine Laboratory, Citadel Hill, Plymouth PL1 2PB, Enqland

Rocky-coast ecosystems; deep-sea ecosystems

Stoecker, D. K. (s)

Horn Point Environmental Laboratories, PO Box 775. Cambridge, MD 21613, USA

Microzooplankton particularly ciliates; secondary production

\section{Stoner, A. W.}

Caribbean Marine Research Center, 805 East 46th Place, Vero Beach, FL 32963, USA

Shallow-water benthic ecology; associations between marine macrofauna and macrophytes; predator-prey relations

\section{Tenore, K. R. (3)}

Chesapeake Biological Laboratory, PO Box 38, Solomons, MD 20688-0038. USA

Benthic ecology and bioenergetics; production; food chains of detritus-based systems

Thayer, G. W. (S

Ecosystem Structure Branch, Southeast Fisheries Center, Beaufort, NC 28516-9722, USA

Wetlands ecology, incl. submerged seagrasses and emergent marshes; production and utilization of detritus

Underwood, A. J. (3)

School of Biol. Sci., Univ. Sydney, Zoology Building, Sydney, NSW 2006. Australia

Experimental intertidal and subtidal ecology; behavior; life cycles of sessile invertebrates

Wangersky, P. J. (s)

SEOS, Univ. of Victoria, PO Box 1700 , Victoria, $\mathrm{BC}$. Canada V8W $2 \mathrm{Y}_{2}$

Organic matter in sea water; theoretical population dynamics; geochemistry; chemical oceanography

\section{Warwick, R. M}

Plymouth Marine Laboratory, Prospect Place West Hoe, Plymouth PL1 3DH, England Structure and functioning of benthic communties; meiofaunal ecology; energy flow

West, J. A.

School of Botany, Univ, of Melbourne, Parkville, VIC 3052, Australia

Reproductive dynamics of red algae; fine structure and parasites of algae.

Williams, $\mathrm{R}$

Plymouth Marine Laboratory, Prospect Place, West Hoe, Plymouth PL1 3DH. England

Plankton ecology; community structure, population dynamics
Wu, B. L.

First Institute of Oceanography. State Oceanic Administration, PO Box 98, Qingdao, People's Republic of China

Ecology and morphology of coelenterates, echinoderms and polvchaetes. Development and reproduction of polychaetes and phoronids

\section{OCEAN MANAGEMENT}

Ahmed, S. I.

School of Oceanography, WB-10, Univ. of Washington, Seattle, WA 98195, USA

Biofouling; application of biotechnology to marine systems

\section{Bernhard, $M$.}

ENEA, PO Box 316, 1-19100 La Spezia, Italy Radioactive and conventional pollution

Brown, B. E.

Dept of Biology, The University, Newcastleupon-Tyne NEI 7 RU, England

Effects of man-made and natural disturbances on coral reefs

Eisler, R.

US Dept of the Interior, Fish and Wildlife Service, Laurel, MD 20708, USA

Toxicology and pollution

Elliott, M. (S)

Dept of Applied Biology, Institute of Estuarine and Coastal Studies, The University, Hull fłu6 7RX, England

Marine pollution assessment and control

Fisher, N. S

Marine Sciences Research Center, State Univ. of New York, Stony Brook, NY 11794-5000, USA Pollotant effects on plankton; radioecology

Fowler, s. W. (s

International Atomic Energy Agency, Marine Environment Laboratory, 19, Ave des Castellans, BP 800, MC-98012 Monaco Cedex pollution effects on zooplankton; radioecology

Gray, J. \$

Institutt for Marinbiologi og Limnologi, Univ. Oslo, Postboks 1064, Blindern. Oslo 3, Norway Pollution effects on benthos animals

Luoma, S. N.

US Dept of the Interior, Geological Survey, Water Resources Div. 345 Middlefield Rd Menlo Park, CA 94025, USA

Bioaccumulation of trace contaminants (principally trace elements and radionuclide waste products)

Mauchline, J.

Scottish Marine Biological Ass, Dunstaffnage Marine Research Laboratory, PO Box 3, Oban, Argyll PA34 4AD, Scotland

Radioactive pollution

McLusky, D. S. ()

Dept of Blological Sclences, Univ, of Stirling. Stirling FK9 4LA, Scotland

Biological effects of pollutants (petro-, chemical, organic enrichment)

Peterson, C. H. (S)

Institute of Marine Sclence, Univ of N. Carolina at Chapel Hill, 3407 Arendell Street, Morehead City, NC 28557, USA

Fisheries management and ecology

Polikarpov, G. G.

Institute of Biology of South Seas, Academy of Sciences Uk. SSR, 2, Nahimov St., Sevastopol 335000, Ukraine

Chemucal ecology, radioecology, radiotracers poliution; protection of sea life

\section{Sherman, K. 1}

US Dept of Commerce, NOAA, Northeast Fisheries Center, 28 Tarzwell Dr., Narragansett, RI 02882, USA

Fisheries ecology; fishery resources; zooplankton

Wu, B. L.

First Institute of Oceanography. State Oceanic Administration, PO Box 98, Qjngdao, People's Republic of China

Effects of pollutants on benthic organisms 